\title{
Diagnosis of schizophrenia
}

Sir-The cautiously euphoric leading article' and the News and Views item ${ }^{2}$ on papers on the localization or non-localization of a susceptibility locus for schizophrenia on chromosome 5 (refs 3.4) merit comment. Despite the extremes of the "anti-psychiatry" tendencies of the 1960 s and 1970s. whose major exponents, Laing and Szasz, you cite in your leading article. no materialist account of the condition(s) diagnosed as schizophrenia could doubt that it (they) must be associated with reasonably specific changes in brain biochemistry and physiology, and that. in particular environments, certain genotypes may well be more likely to express such changes. The problem. as many decades of neurochemical psychiatry has revealed, is to distinguish those changes which are necessarily. sufficiently and exclusively related to the schizophrenic diagnosis. This task is not helped by uncertainties in the diagnosis itself. and the epidemiological evidence which points to its sharply skewed class and ethnic distribution in Europe and the United States today. Hence the relevance of Kennedy et al.'s conclusion that at present it seems that there are different loci, possibly leading to different neurophysiological abnormalities, resulting in a final common pathway "phenotype for schizophrenia" One might better amend this to read in the plural, "phenotypes for schizophrenias" and having done so, to recognize that (a) there is not a one-for-one correspondence between particular genotypes and particular abnormal behaviours such as schizophrenia, and (b) rather than invoke the concept of phenocopy for a phenotypic condition which "mimics" an otherwise genetic condition (as Lander does) it would be better to refer to the genetic conditions which may sometimes be associated with particular phenotypic traits as genocopies.

It is this uncertainty that diminishes the strength of Sherrington et al. 's hope that "it may eventually be possible for psychiatrists to consider genetic counselling in families where chromosome 5 linkage can be reliably established". For genetic counselling to give meaningful advice to such families, it would be necessary to know in how many cases of chromosome 5 linkage schizophrenia does not occur. That is, while Kennedy et al. have shown that such linkage is not necessary for schizophrenia, Sherrington et al. have not shown that it is sufficient - a prerequisite for useful genetic counselling.

Moreover, your leading article and Lander's review accept too easily the view that the genetic studies enable one to reverse the traditional clinical method in which a disorder is diagnosed by its phenotypic (in this case behavioural) manifesta- tions. Instead, you want the genetic measurement to determine whether or not a person is labelled schizophrenic or schizophrenic-sensitive (see Lander's view, contrary to that of Kennedy et al. that it is genetic differences which make possible "splitting schizophrenia into a collection of distinct discases"). This molecular reductionism, reminiscent of the ill-fated dexamethasone suppression test for depression, substitutes biochemistry for behaviour and genetic analysis for psychiatric judgement. It leads you both into a naively neo-darwinian view that. somewhere along the line. there might be adaptive advantages for genes which would otherwise be selectively eliminated, and to conclude that the genetic analysis will make it "feasible to seek means of designing drugs . . . which are more specific and more effective than those available". Your optimism may be justified, but historical precedent suggests that biochemical explanations tend to follow clinical and pharmacological interventions rather than vice versa.

Department of Biology.

STlivi:n Rosi

The Open University,

Walton Hall.

Milton Keynes

$M K 76 A A, U K$

1. Neture 336.95(1908).

L aunder. F.S. Nature 336. $105(1988)$

Sherringlon, R eal Nature 336 f fot (1988).

Kennedy I.L at Narue 336. 167 (1988).

\section{Nobel reaction}

SIR-Your article on the Nobel prize for chemistry (Nature 335, 752; 1988) wrongly suggests that the reaction centre can oxidize water and release oxygen, whereas it is anoxygenic. I should also say that it is incorrect to give the impression that the paper by G. Flemming et al. (Nature 333 . $190 ; 1988)$ was significant in identifying bacteriopheophytin as an electron acceptor. This fact has been known for a very long time based on the studies of many groups in the United States, particularly the work of Parson, Windsor, Holten, Schenck. Dutton and Fajer, as well as Shuvalov and colleagues in the Soviet Union.

\section{AFRC Photosynthesis}

JAMI:S BARIBI:R

Research Group,

Department of Pure

and Applied Biology.

Imperial College,

London SW7 $2 B B, U K$

\section{Radon and smokers}

SIR-There was a calculational error in the estimates of the risk of lung cancer due to radon for smokers and nonsmokers in the recently published BEIR IV report', subject of a news item in your 14 January issue ${ }^{2}$. The modifying term for ange at risk was inadvertently omitted in the program used to prepare Table 2-4 in Chapter 2 and Tables VII-12 to VII-23 in Appendix VII. When this term is included, the estinlated risks for lifetime exposure decline. For example, the corrected estimate of lifetime risk at $1 \mathrm{WLM} / \mathrm{Yr}$ is about 20 per cent smaller for smokers and about 25 per cent smaller for nonsmokers. The risk to exposed smokers relative to similarly exposed nonsmokers therefore becomes slightly larger. 10.5 rather than 10.1. The results for males and females in the general population (without regard to smoking status), presented in Chapter 2 of the report, are not affected by this error.

Corrected tables for smokers and nonsmokers of each sex can be obtained from the board at the address below.

WILIIAM H. EIII:T"

Board on Radiation Effects Research

National Research Council.

2101 Constitution Avenue,

Washington DC 20418, USA

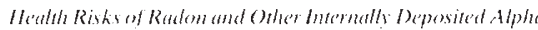 Partich Eminers: BEIR IV (National Academy Press. Washington lesis)
2. Palca. J. Nottitre 331, 107 (1988)

\section{Virulence opposition}

SIR-All societies have their pariahs and the scientific society is no exception. We should promote research and science that is in mankind's best interests and condemn that which is contrary.

The letter entitled "Increased virulence of Yersinia pseudotuberculosis by two independent mutations" (Nature 334. $522-525 ; 1988$ ) shows that introduction of mutations in both the invasin gene and the Yopl gene enhanced virulence of $Y$. pseudotuberculosis vis-à-vis the wild type; individual gene mutations diminished the virulence. $Y$. pestis causes plague. The authors introduced the functional Yopl gene into $Y$. pestis and diminished its infectivity.

This work was carried out by members of the Swedish Defense Research Establishment. Sweden is a major supplier of arms to developing countries. It would be naive to think that the work was supported because it wanted to diminish the infectivity of the plague bacillus. When the Swedish Defence Establishment succeeds in introducing the correct form of the invasin gene as well as the Yopl gene into $Y$. pestis, thereby producing a superplague bacillus, do you think it will brag about it in the pages of Nature?

JACK A. KORNBI.ATT

Biology, Concordia University,

1455 de Maisonneuve.

Montreal, $P Q$,

Canada $H 3 G I M 8$ 